https://doi.org/10.35336/VA-2021-E-51-53

\title{
CRYOBALLOON PULMONARY VEIN ISOLATION IN A PATIENT \\ WITH PAROXYSMAL ATRIAL FIBRILLATION AFTER ATRIAL SEPTAL DEFECT CLOSURE \\ USING THE “AMPLATZER” OCCLUDER
}

I.A.Taymasova, M.V.Yashkov, M.V.Kadirova, E.A.Artyukhina

A.V.Vishnevskiy National Medical Research Center of Surgery, Russia, Moscow, 27 Bolshaya Serpukhovskaya str.

A case report of cryoballoon ablation for atrial fibrillation in a patient after atrial septal defect closure is presented.

Key words: atrial septal defect; occlude; atrial fibrillation; cryoballoon isolation

Conflict of Interests: nothing to declare.

Received: 26.03.2020 Revision Received: 11.04.2020 Accepted: 08.06.2020

Corresponding author: Irina Taymasova, E-mail: irina-tame@yandex.ru

For citation: Taymasova IA, Yashkov MV, Kadirova M, Artyukhina EA. Cryoballoon pulmonary vein isolation in a patient with paroxysmal atrial fibrillation after atrial septal defect closure using the "Amplatzer" occluder. Journal of Arrhythmology. 2020;28(E): 51-53. https://doi.org/10.35336/VA-2021-E-51-53.

Atrial septal defect (ASD) accounts for $30-40 \%$ of all cases of congenital heart defects in adults. ASD is commonly accompanied by the volume overload of the right atrium (RA) and right ventricle (RV) promoting alterations in the left atrium (LA) electrophysiological properties. These alterations underlie the development of heart rhythm disturbances [1]. ASD is treated either by open-heart surgery or by an endovascular approach using various occluder devices. Atrial fibrillation (AF) occurs in $25 \%$ of patients with ASD before surgery and $60 \%$ of patients in the long-term period after surgery [2, 3]. Interventional treatment of AF is effective in patient's refractory to antiarrhythmic therapy [4]. Since interventional procedures require access to the LA, an implanted occluder limits the manipulations of operators during the intervention. We aim to report an effective and safe method of treating paroxysmal AF in the patient after endovascular ASD closure with the "Amplatzer" occluder.

A patient was admitted to the hospital with paroxysmal atrial fibrillation, a tachysystolic pattern $\left(\mathrm{CH}_{2} \mathrm{DS} \mathrm{S}_{2}\right.$ VASc score of 1, HAS-BLED score of 0). EHRA III. The patient had secondary ASD closed with the "Amplatzer" occluder in 2001. The patient had concomitant NYHA class 1 chronic heart failure. According to transthoracic echocardiography (TTE), the RA and LA were enlarged. There was no dislocation of the occluder and both discs were deployed. There were no blood shunts across the atrial septum. Multislice computed tomography (MSCT) of the $L A$ and pulmonary veins $(P V)$ reported an $L A$ volume of $105 \mathrm{~mL}$. The venous connection was without any alterations and the occluder was visualized along the entire atrial septum (Fig. 1).

To minimize the number of surgical accesses to the $L A$ and considering the presence of previously implanted occluder, we performed cryoballoon PV isolation.

The Seldinger technique was employed to perform the venous access under local anesthesia in the cath-lab. A decapolar electrode was positioned through the left subclavian vein into the coronary sinus. A quadropolar electrode for temporary stimulation was positioned through the left femoral vein into the right ventricular cavity. The Swartz introducer sheath with a transseptal needle was passed through the right femoral vein into the RA cavity.

Under transesophageal echocardiography (TEE) (Fig. 2), the atrial septum and the implanted occluder were visualized. We evaluated the atrial septum and selected the free area of the posterior inferior margin of the occluder to perform the transseptal puncture under TEE monitoring.

After the access to the LA was achieved, we performed cryoballoon PV isolation according to the standard protocol. The Swartz introducer was replaced with a FlexCath Advance controlled delivery system, followed by positioning of the cryoballoon and multipolar circular electrode in each PV. Additionally, 3D reconstruction of the LA anatomy was performed using the Astrocard Navigation System (Russian Federation) to achieve better visualization of the PV and construct voltage maps before and after PV isolation. Before isolation, spike activity in each

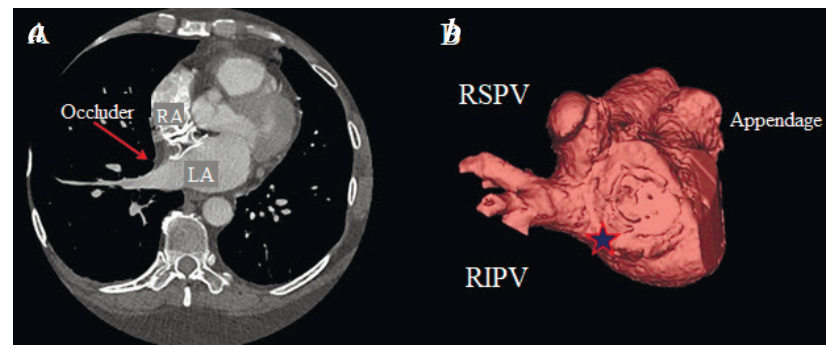

Fig.1. MSCT findings: a) Axial plane - left atrial level: the atrial septum with the implanted device; b) $3 D$ reconstruction of the $L A$ from side of the atrial septum, the occluder's shadow is visualized. The asterisk denotes the native area of the atrial septum suitable for the transseptal puncture. $R A$ - right atrium, LA - left atrium, RSPV - right superior pulmonary vein, RIPV - right inferior pulmonary vein. 
$P V$ was assessed. After the balloon inflation, optimal PV occlusion was assessed by contrast injection. Cryoapplication time was 180 seconds and then was followed by cooling and deflation of the balloon. The minimum temperature in the upper left $P V$ and the lower left $P V$ was $55^{\circ} \mathrm{C}$ and $42^{\circ} \mathrm{C}$, respectively. Before cryoablation of the right $P V s$, the quadropolar electrode was positioned in the superior vena cava to stimulate the phrenic nerve. The minimum temperature in the upper right $P V$ and the lower right $P V$ was $-44{ }^{\circ} \mathrm{C}$ and $-50{ }^{\circ} \mathrm{C}$, respectively (Fig. 3). The PV was successfully isolated when the spike activity was verified by the multipolar circular electrode recordings. There were no technical difficulties associated with balloon positioning. The procedure lasted for 110 minutes and the fluoroscopy time was 20 minutes.

There were no complications in the postoperative period. The control TTE was performed after 2 hours of the intervention, and days 1 and 2 confirmed the absence of any devices damages and blood shunts across the atrial septum. The patient was discharged 2 days after the intervention.

\section{DISCUSSION}

There are few studies on treating AF in patients after endovascular ASD closure. Santangeli et al. [5] reported the outcomes of 39 patients with previously implanted occluders treated for AF. Of them, 33\% of patients had paroxysmal AF, $51 \%$ - persistent AF, and 16\% - long-term persistent AF. The transseptal puncture in the area of the native interatrial septum was performed in 35 patients. Four patients required the puncture to be performed through an occluder. When the introducer and the needle were positioned in the center of the occluder, the traction was performed. After contrast injection, the needle was replaced with a guide, and the balloon was delivered to the occluder. The puncture site was dilated and then the introducer and electrodes were inserted. Control TTE did not show any shunts across the atrial septum.

Sang et al. [6] presented a single-center experience of 16 catheter ablation procedures in patients with previously implanted occluder devices. Importantly, the operators did not use additional TEE or intracardiac ECHO control. To achieve better visualization before the transseptal puncture, the contrast was injected to evaluate the atrial septum and the implantation site of the occluder relative to the great arteries and heart valves in different planes. If the transseptal puncture in the native atrial septum area failed, the central zone of the occluder was punctured. The balloon was used to pass the introducer into the LA. Li et al. [7] reported 9 cases of catheter ablation after endovascular ASD closure. Eight patients underwent the transseptal puncture under X-ray control and 1 patient underwent intraoperative TEE. The operators performed the access to the LA along the posterior inferior margin of the occluder; in case of unsuccessful attempts, the transsep- tal puncture was performed through the occluder. But it increased both, the duration of fluoroscopy and the time of procedure itself. Revishvili et al. [8] reported a clinical case of a transseptal puncture approach in the occluder-free zone of the atrial septum in a patient after endovascular ASD closure. The transseptal puncture was performed under intracardiac ECHO control.

The outcomes of catheter ablations in patients after endovascular ASD closure and the rate of postoperative

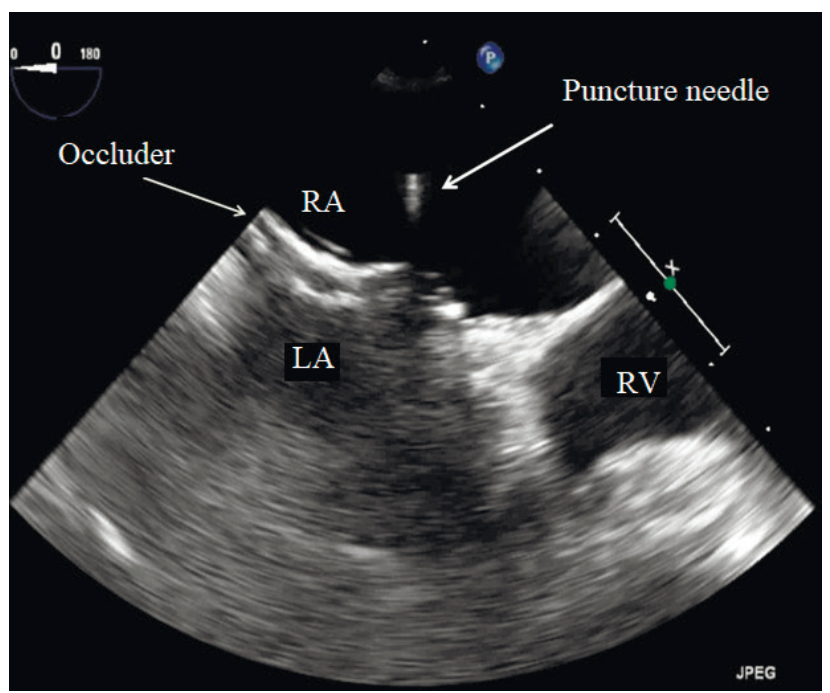

Fig. 2. The transseptal puncture under TEE monitoring: the heart chambers, the implanted occluder, the atrial septum, the puncture needle is visualized. TEE transesophageal echocardiography, $R A$ - right atrium, LA - left atrium, $R V$ - right ventricle

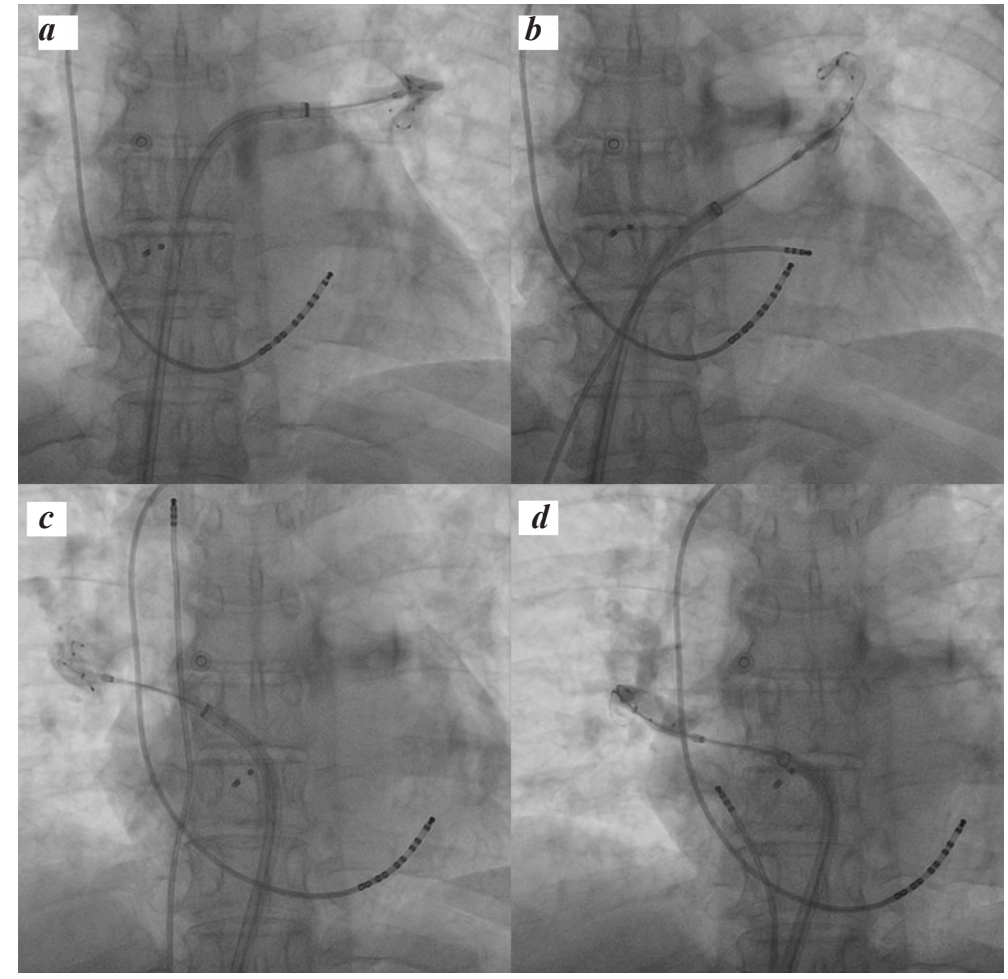

Fig. 3. a) Cryoballoon ablation of the LSPV; b) Cryoballoon ablation of the LIPV; c) Cryoballoon ablation of the RSPV; d) Cryoballoon ablation of the RIPV. A frontal plane. The balloon in the PV and the shadow of the occluder are visualized. LSPV - left superior pulmonary vein, LIPV - left inferior pulmonary vein, RSPV - right superior pulmonary vein, RIPV - right inferior pulmonary vein 
complications did not differ from those reported for patients with an intact atrial septum.

\section{CONCLUSION}

Cryoballoon PV isolation can be safely performed in patients with previously implanted occluder devices in the atrial septum. The site of access in the LA does not complicate the manipulation of instruments in the LA and does not affect the positioning of the cryoballoon in the LA. Preoperative MSCT of the LA allows evaluating the size and position of the occluder in the atrial septum. Intraoperative TEE allows selecting a safe, occluder-free zone to perform the transseptal puncture.

\section{REFERENCES}

1. Spies C, Khandelwal A, Timmermanns I, et al. Incidence of atrial fibrillation following transcatheter closure of atrial septal defects in adults. American Journal of Cardiology. 2008;102 902-906. https://doi.org/ 10.1016/j.amjcard.2008.05.045

2. Donti A, Bonvinci M, Placci A, et al. Surgical treatment of secundum atrial septal defects in patients older than 50 years. Italian Heart Journal. 2001;2: 428-432. PMID: 11453578.

3. Berger F, Ewert P, Bjornstad PG, et al. Transcatheter closure as standard treatment for most interatrial defects: experience in 200 patients treated with the Amplatzer septal occluder. Cardiology Young. 1999;9: 468-473. https:// doi.org/ 10.1017/s1047951100005369.

4. Kirchhof P, Benussi S, Kotecha D, et al. 2016 ESC Guidelines for the management of atrial fibrillation developed in collaboration with EACTS. European Heart Journal. 2016;37(38): 2893-2962. https://doi.org/10.1093/eurheartj/ehw210.
5. Santangeli P, Di Biase L, Burkhardt JD, et al. Transseptal access and atrial fibrillation ablation guided by intracardiac echocardiography in patients with atrial septal closure devices. Heart Rhythm. 2011;8(11): 1669-1675. https:// doi.org/10.1016/j.hrthm.2011.06.023.

6. Sang C-H, Dong J-Z, Long D-Y, et al. Transseptal puncture and catheter ablation of atrial fibrillation in patients with atrial septal occluder: initial experience of a single centre. Europace. 2017;10(1): 101-107. https://doi.org/10.1093/europace/eux282. 7. Li X, Wissner E, Kamioka M, et al. Safety and feasibility of transseptal puncture for atrial fibrillation ablation in patients with atrial septal defect closure devices. Heart Rhythm. 2014; 11(2): 330-335. https://doi.org/10.1016/j.hrthm.2013.11.011 8. Revishvili ASh, Rzaev FG, Sopov OV, et al. Interventional treatment of atrial fibrillation using intracardiac ultrasound in patient with bilateral mini-invasive thoracoscopic radiofrequency isolation of pulmonary veins and atrial septal closure with "Amplatzer" occluder. Annals of arrhythmology. 2008;4: 70-78. (In Russ.). 